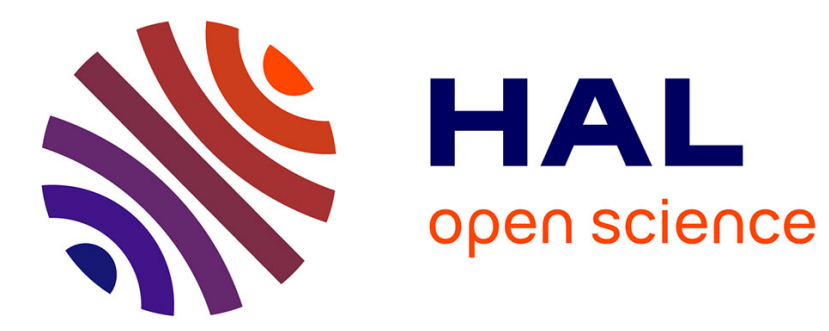

\title{
Electrical resistivity of the Kondo lattice
}

\author{
M. Lavagna, Claudine Lacroix, Michel Cyrot
}

\section{To cite this version:}

M. Lavagna, Claudine Lacroix, Michel Cyrot. Electrical resistivity of the Kondo lattice. Journal of Applied Physics, 1982, 53 (3), pp.2055-2057. 10.1063/1.330742 . hal-01896473

\section{HAL Id: hal-01896473 https://hal.science/hal-01896473}

Submitted on 16 Oct 2018

HAL is a multi-disciplinary open access archive for the deposit and dissemination of scientific research documents, whether they are published or not. The documents may come from teaching and research institutions in France or abroad, or from public or private research centers.
L'archive ouverte pluridisciplinaire HAL, est destinée au dépôt et à la diffusion de documents scientifiques de niveau recherche, publiés ou non, émanant des établissements d'enseignement et de recherche français ou étrangers, des laboratoires publics ou privés. 


\section{Electrical resistivity of the Kondo lattice}

M. Lavagna, C. Lacroix, and M. Cyrot

Citation: Journal of Applied Physics 53, 2055 (1982); doi: 10.1063/1.330742

View online: https://doi.org/10.1063/1.330742

View Table of Contents: http://aip.scitation.org/toc/jap/53/3

Published by the American Institute of Physics

\section{Articles you may be interested in}

Theory of Kondo lattice

Journal of Applied Physics 50, 7555 (1979); 10.1063/1.326898

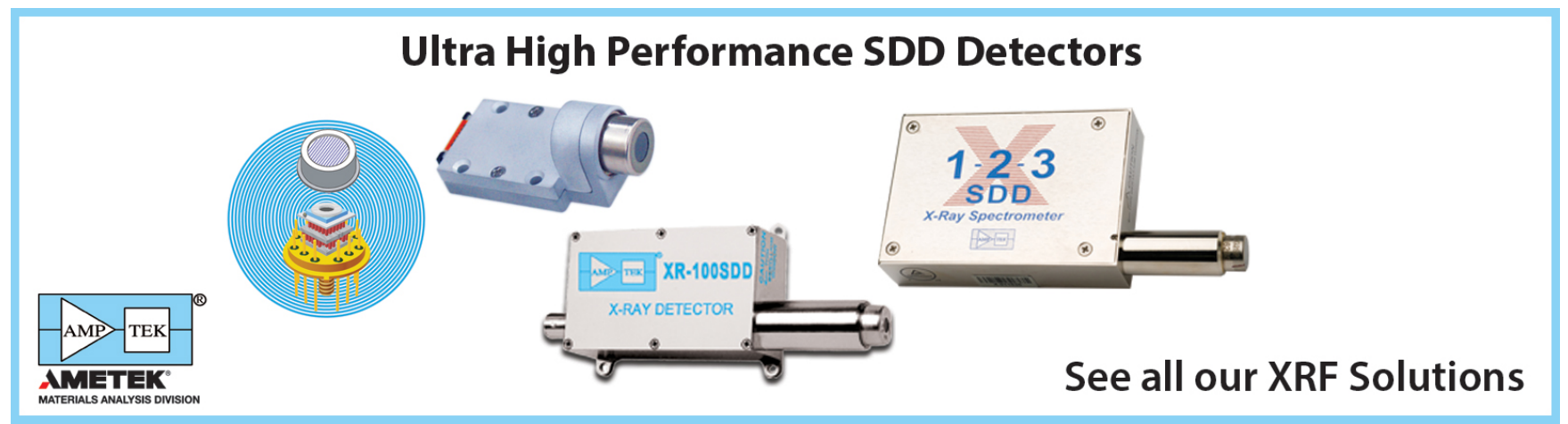




\title{
Electrical resistivity of the Kondo lattice
}

\author{
M. Lavagna, C. Lacroix, and M. Cyrot
}

Laboratoire Louis Néel, CNRS associé à l'Université Scientifique et Médicale de Grenoble, 166X, 38042 Grenoble-Cédex, France

The electrical resistivity of the Kondo lattice is studied within the model of Lacroix and Cyrot ${ }^{1}$ : In this model the Kondo fluctuations are described by a ficticious s.f. hybridization $J \mathbf{x} / 2$, where $\mathrm{x}$ is determined selfconsistently. Thermal and spatial fluctuations of the parameter $\mathrm{x}$ are found to play an important role in the transport properties. At low temperatures the resistivity depends strongly on the conduction electron number $\mathrm{n}$ : We find for $\mathrm{n}<1$ a metallic behavior with a linear increase of the resistivity and a maximum at a temperature $T_{1} \sim(1-n)^{1 / 3} T_{k}$. For $n=1$ the resistivity is that of a semiconductor: $\pi \sim \exp (T K / T)$. At higher temperature (above $T_{x}$ ) the system may be treated as a collection of incoherent Kondo impurities and the resistivity exhibits a logarithmic decrease as can be expected.

PACS numbers: 72.15.Qm

\section{I-INTRODUCTION}

The concentrated compounds of anomalous rare earth exhibit two kinds of transport properties (2). Most ambivalent Cerium and Ytterbium based compounds ( $\mathrm{CeAl}_{2}$, $\mathrm{CeIn}_{3}, \mathrm{CePd}$ ) behave as a metal at low temperatures with an increasing resistivity when $\mathrm{I}$ arises from $0 \mathrm{~K}$ : the resistivity passes through a maximum at $T_{\max }$ before exhtbiting the Kondo decrease going as $\mathrm{Ln} I$ at high temperature. On the other hand, Samarium and Thulium compounds ( $\mathrm{Sm}_{6}$, ImSe and probably $\mathrm{N}-\mathrm{SmS}$ ) are typical of small gap semi-conductors with infinite resistivities at $T=O K$ followed by a large logarithmic decrease with temperature. A particularly interesting case is provided by $\mathrm{Tm}_{\mathrm{x}} \mathrm{Se}$, which depending upon its stoechlometry belongs either to the first or the second category.

While all the theories made so far concerned the mixed valences $(3,4,5)$, it seemed interesting to see what the Kondo lattice model gives for the transport properties. The solution to the problem depends on the range of temperature : at high temperatures, interactions between rare earth atoms become negligible and the system may be treated as a collection of Incoherent impurities; at low temperature, coherence develops between virtual bound states and the material behaves as a Kondo lattice.

The model used here to describe the Kondo lattice is that of Lacrolx and Cyrot (1) who transform the Kondo interaction into a fictitious $s-f$ hybridization. Thermal and spatial fluctuations are found to play an essential role in the description of the transport properties. The collection of impurities is treated within the phase shift method.

\section{II-REVIEW OF THE KONDO LATTICE MODEL}

The Kondo lattice model was first introduced by Doniach (7) as a generalization of the Kondo Hamiltonian to the concentrated case. The essential feature lies in the competition between the Kondo effect and magnetic order. In a mean-field approximation on a one-dimensional lattice, Doniach found that the ground state is antiferromagnetic for low $\mid J / / D$ and is a non magnet1c Kondo singlet for large $\mid \mathrm{J} / / \mathrm{D}$. These results were confirmed by Jullien et al (8) using a renormalization group treatment.

Lacroix and Cyrot (1) generalized to the Kondo lattice a method of functional integration first introduced by Yoshimori and Sakural (6) for the simple impurity case. Using this method, they transformed the Kondo interaction into a fictitious s-f hybridization. The Hamiltonian obtained is :

$$
\begin{aligned}
\mathrm{H}= & \sum_{\mathrm{k} \delta}\left(\varepsilon_{\mathrm{k}}+\mathrm{J} / 4\right) \mathrm{C}_{\mathrm{k} \sigma}^{+} \mathrm{C}_{\mathrm{k} \sigma}+\sum_{1 \sigma}\left(\mathrm{E}_{0}+\mathrm{Jn} / 4\right) \mathrm{d}_{1 \sigma}^{+} \mathrm{d} i \sigma \\
& +\frac{\mathrm{J}}{2} \sum_{i} \mathrm{x}_{i}\left(\mathrm{~d}_{i \uparrow}^{+} \mathrm{C}_{1 \uparrow}+\mathrm{C}_{i \downarrow}^{+} \mathrm{d}_{i \downarrow}\right)+\frac{\mathrm{J}}{2} \sum_{1} \mathrm{y}_{i}\left(\mathrm{~d}_{i \downarrow}^{+} \mathrm{C}_{1 \downarrow}+\mathrm{C}_{i \uparrow}^{+} \mathrm{d}_{1 \uparrow}\right)
\end{aligned}
$$

$n$ is the number of conduction electrons per atom (knowing that there is one impurfty per site),

$x_{i}$ and $y_{i}$ represent the fictitious $s-f$ hybridization and are determined selfconsistently. They can be considered as an order parameter : their average values are reduced to zero above a temperature $\mathrm{T}_{k}^{+1}$. In the following $x_{1}$ will be taken equal to $y_{i}$ and uniform ie $x_{1}=y_{1}=x$.

With a rectanguiar conduction band of width $2 \mathrm{D}$, they obtained for the density of states of the $4 \mathrm{f}$ and conduction electrons :

$$
\rho_{d}(\omega)=\frac{1}{8 D} \frac{J^{2} x^{2}}{\left(\omega-E_{0}-\frac{J \pi}{4}\right)^{2}} \quad ; \quad \rho_{c}(\omega)=\frac{1}{2 D}
$$

when $\omega_{1}<\omega<\omega_{2}$ and $\omega_{3}<\omega<\omega_{4}$ and $\rho_{d}=\rho_{0}=0$ overwise; the expressions of $\omega_{1}, w_{2}, \omega_{3}, \omega_{4}^{4}$ are given otherwhere (1).

The Kondo temperature $I_{X}{ }^{1}$ given by the ground state energy has the usual varlation $\mathrm{De} / \rho \mathrm{\rho J}$, where $\rho$ is the initial density of states at the Fermi level as for a single impurity. The schematic behaviour of $\rho_{d}$ and $\rho_{c}$ is shown in figure 1. As soon as $x \neq 0$, a gap ls opened in the density of states of the order of $T_{R}$ around the energy of the impurity level: the width of the reso-

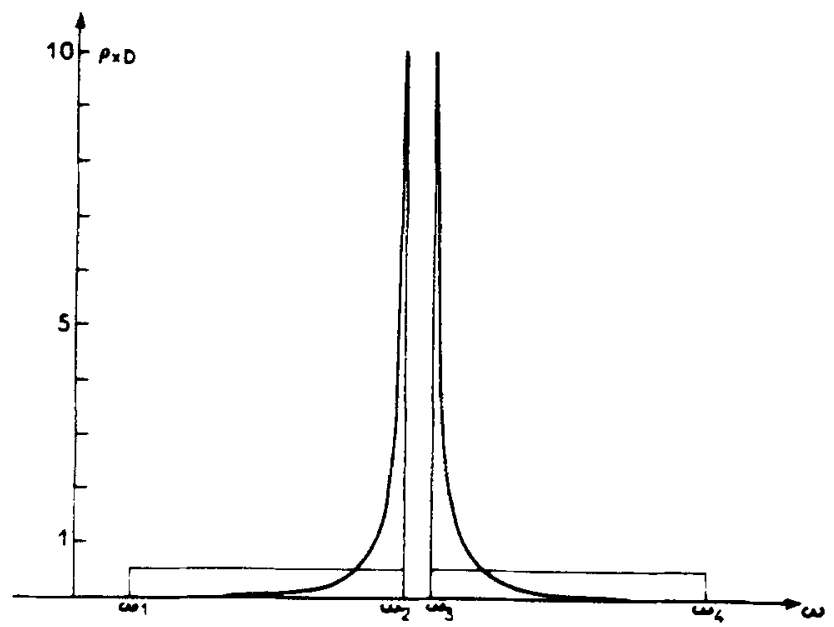

Eigure 1 : Schematic density of states of conduction and f electrons for the Kondo lattice. 
nance is of the same order. The phase diagram is discussed as a function of $J / D$ and $n$ : the system is magne$t 1 c$ under a critical value of $\mathrm{J} / \mathrm{D}$; the magnetic order is then ferromagnetic for low values of $\mathrm{n}$ and becomes antiferromagnetic when $n$ is close to 1 . The ground state is found to be insulating when the conduction band is half-fllled : $n=l$.

In a mean-fleld theory, as soon as temperature gets larger then $T_{K}^{+}, x$ and $y$ are reduced to zero and the resistivity obtained is that of free electrons. In fact, experiments show that the resistivity exhibits a large decrease from $5 \mathrm{~K}$ to 100 or $200 \mathrm{~K}$, well above $\mathrm{T}_{\mathrm{K}}^{+}$ ( 5 or 10K). To account for this, we must go beyond mean-field theory and introduce thermal fluctuations. The vartation of the free energy $F$ as a function of $x$ is characteristic of a second order transition with the presence of a minimum for temperatures lower than $\mathrm{I}_{\mathrm{K}}^{+}$. colng beyond mean-field theory leads us to consider

a statistic distribution around the value of the minimum. written:

- In low temperature limit, the free energy can be

$$
F=-\frac{J x^{2}}{2}\left[1-\frac{J}{2 D}\left(-1+\operatorname{Ln} \frac{J^{2} x^{2}}{4 n D^{2}}\right)\right]
$$

exhibiting a minimum for $\frac{\mathrm{J}^{2} \mathrm{x}^{2}}{\mathrm{D}^{2}}=4 \mathrm{n} \mathrm{e}^{2 \mathrm{D} / \mathrm{J}}$ as found by

- In the high temperature limit:

$$
F=\frac{J^{2} x^{2}}{4 D} \text { In } \frac{T}{I_{K}^{+}}
$$

$$
\text { w1th }\left\{\begin{array}{l}
\mathrm{kT}_{\mathrm{K}}^{+}=\mathrm{a}(\mathrm{n}) \mathrm{De} \mathrm{e}^{1 / \rho \mathrm{J}} \\
\text { and } \mathrm{a}(\mathrm{n})=1.176 \sqrt{\mathrm{n(2-n)} \mathrm{e}^{1-\frac{1-n}{2-\pi}}}
\end{array}\right.
$$

The high temperature value of $\left\langle x^{2}\right\rangle$ is given by :

$$
\left\langle\mathrm{x}^{2}\right\rangle=\frac{\mathrm{kT}}{\frac{\mathrm{J}^{2}}{2 \mathrm{D}} \operatorname{Ln} \frac{\mathrm{T}}{\mathrm{T}_{\mathrm{K}}^{+}}}
$$

Effectively, the thermal fluctuations are found to be of great importance above $\mathrm{T}_{\mathrm{K}}^{+}$and can considerab1y modify the resistivity behaviour at high temperature.

\section{III-RESISTIVITY OF THE COLIECTION OF IMPURITIES AT HIG \\ IEMPERATURES}

At high temperatures, all the virtual bound states scater the conduction electrons in a completely incoherent way. The system can be considered as a collection of incoherent impurities. Thermal fluctuations and resistivity are then calculated within the classical phase shift method.

- In order to evaluate the thermal fluctuations we start from the phase shift $\phi(\omega)$ introduced by the impurity $(9,10)$ :

$$
\Phi(\omega)=\operatorname{Arctg} \frac{\Delta}{\omega}
$$

where $\Delta$ is the width of the virtual bound state :

$$
\Delta=\pi\left(\frac{\mathrm{Jx}^{2}}{2}\right) \frac{1}{2 \mathrm{D}}
$$

The free energy can be written :

1 We distinguish in this paper the temperature $\mathrm{T}_{\mathrm{K}}^{+}$above which the Kondo parameters $x$ and $y$ are equal to zero, and the temperature $T_{K}$ given by the ground state energy. The two temperatures are in fact of the same order.

$$
F=-\frac{J x^{2}}{2}+\frac{2}{\pi} \int_{-D}^{+D} f(\omega) \operatorname{Arctg} \frac{\Delta}{\omega} d \omega
$$

At high temperatures, the result is similar to the high temperature limit of the Kondo lattice :

$$
\left\{\begin{array}{l}
F=\frac{J^{2} x^{2}}{4 D} \operatorname{In} \frac{T}{T_{K}^{+}} \\
\text {with } \mathrm{kT}_{\mathrm{K}}^{+}=1,14 \mathrm{De}^{20 / J}
\end{array}\right.
$$

- Considering that the potential responsible for the scattering of the conduction electrons is the fictitious s-f hybridization Jx , the resistivity is then calculated within the 2 phase shift method $(9,10)$ :

$$
\begin{aligned}
& \rho(\omega)=\left(\frac{4 \pi}{n e^{2} k_{F}}\right) \sin ^{2} \Phi(\omega) \\
& \rho=\left(\frac{4 \pi}{n e^{2} k_{F}}\right) \frac{3 J^{4} x^{4}}{16 D^{2}(k T)^{2}}
\end{aligned}
$$

Taking account of thermal fluctuations to evaluate $x^{4}$, the resistivity behaves at high temperatures as :

$$
\rho=\left(\frac{4 \pi}{n^{2} k_{F}}\right) \frac{9}{4 \operatorname{Ln}^{2} \frac{T}{T_{K}}}
$$

and for $\mathrm{T} \gg \mathrm{T}_{\mathrm{K}}$ :

$$
\rho=\left(\frac{4 \pi}{n e^{2} k_{F}}\right) \frac{9}{4}\left(\frac{J}{2 D}\right)^{2}\left[1+\frac{J}{D} \ln \frac{k r}{D}\right]
$$

The resistivity exhibits a Kondo behaviour at high temperature with a logarithmic decrease in $\mathrm{J}$ in $\mathrm{kT} / \mathrm{D}$. This provides the explaination of the high temperature behaviour of many concentrated compounds of anomalous rare earths. In fact, the resistivity does not reach the zero value at high temperature due to the effects of phonons and impurities.

\section{IV-RESISTIVITY OF THE KONDO LATTICE AT LOW TEMPERATURES}

At low temperatures, coherence develops between the 1mpurities and the system may be described as a Kondo lattice. If the Kondo parameter $x$ is unfform on all the sites, the Hamlltonian ( 1 ) can easily be diagonalized and nothing else is able to scatter electrons. On the other hand, if we consider the spatial fluctuations (from site to site) of the $a-f$ hybridization, it remains the following potential:

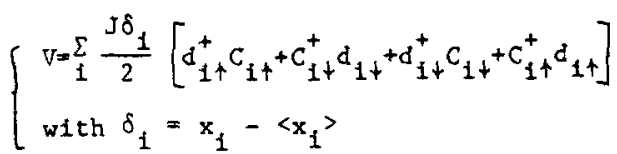

Among all the posstble mechanisms (phonons, ...), spatial fluctuations are the mechanism retained here to explain the electron scattering. Let us remark that $\left\langle x_{1}\right\rangle=0$ at high temperature and the potentiel $V$ is the same as that considered in the previous section.

The relaxation time is calculated by means of the I-matrix related to $\mathrm{V}$ :

$$
\tau_{i}(\omega)^{-1}=-I m T_{s s}
$$

(ss and dd conduction electrons are negligible)

If we express $I_{s s}$ as a function of the Green's function, we find :

$$
\tau_{i}(\omega)^{-1}=\left(\frac{J \delta_{i}}{2}\right) \rho_{d}(\omega)
$$


While all the calculations, up to now, have been carried out successfully with a rectangular conduction band, It appears that, for transport properties, such a band does not give a continuous passage from metals to insulator when $n$ tends to 1 , due to a discontinuous variation of $\rho\left(E_{F}\right)$ when $E_{F} \rightarrow w_{2}$ (figure 1$)$. To overcome this difficulty, an elliptic band is taken in the following. The details of the determination of parameters for the elliptic band are presented elsewhere (11).

\section{$1-$ Res}

The expression of the conductivity is given by :

$$
\sigma=\frac{2}{3} e^{2} \int_{\omega_{1}, \omega_{3}}^{\omega_{2}, \omega_{4}}\left(-\frac{d f}{d \omega}\right) \rho_{c}(\omega) v^{2} \tau d \omega
$$

For $n$ close to 1 , only the states near $\omega_{2}$ are to consider for the resistivity. For these states, the band is quasi-parabolic and we can write for $\omega$ near $\omega_{2}: \omega_{2}-\omega=\frac{1}{2} m{ }^{*} v^{2}$

$$
\sigma=\sigma(0)\left[1+\left(\frac{\mathrm{T}}{\mathrm{T}_{1}}\right)^{2}\right]
$$

At very low temperature $\left(T<T_{x} / 3\right),\left\langle x^{2}\right\rangle$ is not very different of $x^{2}$ in $(T=0)$ and we can replace $x$ by its minimum value at $\mathrm{T}=0 \mathrm{~K}$. In this way, we find :

$\sigma(0)=\frac{2 e^{2}}{3 m^{*} D}-\frac{4}{J^{2} \delta^{2}}\left[\frac{3 \pi}{4}(1-n)\right]^{2 / 3}\left(\frac{J^{2} x^{2}}{4 D}\right)$

We recognize in this expression the classical formula $\mathrm{N}_{\mathrm{h}} \mathrm{e}^{2} \mathrm{t} / \mathrm{m}$ obtained every time the electron behave as complecely free. Knowing that $\left\langle\delta^{2}\right\rangle$ is in $\mathrm{kT} / 2|\mathrm{~J}|$, this term gives a linear contribution to the resistivity:

$\rho(0)=\left[\frac{3 D}{2(1-n)^{2} T_{K}^{4}}\right]^{1 / 3} \frac{1}{2 e^{2}}|J| \frac{T}{T_{K}}$

The expression of $\mathrm{T}_{1}$ is :

$$
\mathrm{T}_{1}=0,367 \mathrm{~T}_{\mathrm{K}}(1-\mathrm{n})^{1 / 3}
$$

The resistivity exhibits a maximum at the same temperature $T_{1}$. The thermal variation of the resistivity at low temperature and for $n \neq 1$ is represented in figure 2 . It is of metallic type with an increase of the resistivity with increasing temperature. The temperature at which the resistivity passes through a maximum is of the order of $(1-n)^{1 / 3} T_{4}$. The value of the raximum varies then as $1 /(1-n)^{1 / 3}$, becoming infinite for $n=1$.

\section{2-Resistivivity_tn_the semiconductor_case ( $\mathrm{nx} 1$ )}

$E$ and $E_{2}$ take respectively the values 0 and $J / 4$ $\left(\mathrm{E}_{\mathrm{F}}\right.$ at ${ }^{\circ}$ the middle of the gap). The result is:

$$
\sigma=\frac{16 \mathrm{e}^{2}}{3 \mathrm{~m}^{*}} \frac{\mathrm{kT}}{\mathrm{D}} \frac{\mathrm{J}^{2} \mathrm{x}^{2}}{4 \mathrm{D}} \frac{\mathrm{e}^{-\beta \frac{\mathrm{J}^{2} \mathrm{x}^{2}}{4 \mathrm{D}}}}{\left(\frac{\mathrm{J} \delta}{2}\right)^{2}}
$$

The thermal variation of the reststivity is of semi-conductor type (Infinite value at $T=0$ ) with an exponential decrease in $e^{\mathrm{TR}} / \mathrm{T}$. Figure 2 shows the resistivity behaviour at low temperature in the semi-conductor case.

Ve report in the same figure the results obtained for intermediate temperatures by interpolation calculations on computer.

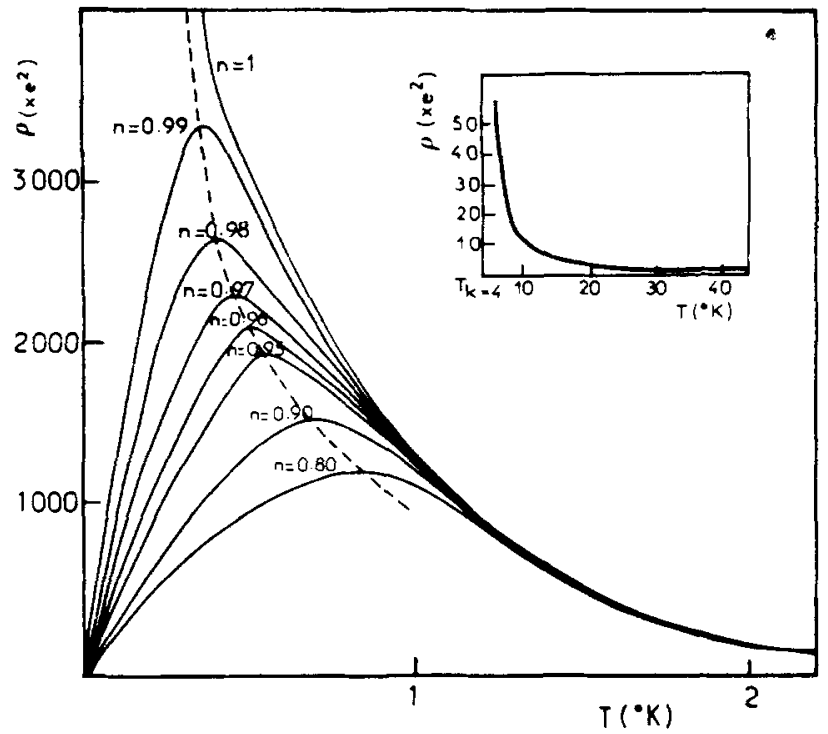

Figure 2 : Temperature dependance of resistivity for various values of $n(J=0.2 \mathrm{eV}$ and $\mathrm{D}=1 \mathrm{eV})$

\section{V-CONCIUSION}

Finally, this work permits us to show that a good description of the concentrated systems at anomalous rare earth is provided by the collection of impurities at high temperatures and by the Kondo lattice at low temperatures. It points out too the importance of thermal and spatial fluctuations to understand the mechanism of electron scattering. The result obtained is a logarithmic decrease of the resistivity at high temperature and a behaviour elther of metallic or semiconductor type at low temperature according to the value of $n$. In the metallic case $(n \neq 1)$, the resistivity increases with $T$ and passes through, maximum at a temperature of the order of $T_{4}(1-n) 1 / 3$. In the semiconductor case $(n=1)$, the reststivity is found to be in $\mathrm{e}^{\mathrm{T}_{\mathrm{K}}} / \mathrm{T}$.

\section{RE FERENCES}

1. Lacrolx C., CYROT M., Phys. Rev. B20, 1969 (1979)

2. Lawrence J.M., Riseborough P.S., Parks R.D., Report on Progress in Physics (1981)

3. Ghatak S.K., Avignon M., Bennemann K.H., J. Phys. F 6, 1441 (1976)

4. Entel P., Mühlschlegel B., Ono Y., Z. Physik B 38 , 227 (1980)

5. Coqblin B., Bhattacharjee A.K., Jullien R., Journal of Magn. Magn., Mat. 15-18, 995 (1980)

6. Yoshimori A., Sakurai A., Prog. Theor. Phys., Supp1. 46, 162 (1970)

7. Doniach S., Physica, B91, 231 (1977)

8. Jullien R., Fields J.N., Doniach S., Phys. Rev. B16, 4889 (1977)

9. Friedel J., Adv. Phys. 3 , 446 (1954)

10. Frledel J., Nuovo-Cimento, Supp1. I, 287 (1958)

11. Lavagna M., Lacroix C., Cyrot M., J. Phys. F (to be published) 